\title{
INTRASPECIFIC AGGREGATION DECREASES LOCAL SPECIES DIVERSITY OF ARTHROPODS
}

\author{
Joseph A. Veech, ${ }^{1}$ Thomas O. Crist, and Keith S. Summerville ${ }^{2}$ \\ Department of Zoology, Miami University, Oxford, Ohio 45056-1400 USA
}

\begin{abstract}
The aggregation model of species coexistence predicts that insect species diversity within a community is maintained by intraspecific aggregation among resource patches. An untested corollary of this prediction is that diversity within resource patches should decrease with increasing intraspecific aggregation. The recently derived speciesaggregation relationship provides a general formulation of this prediction: as intraspecific aggregation increases within a geographic area, the species richness within samples of the area decreases. We tested this prediction by compiling and analyzing 76 data sets of arthropod species distribution and abundance. For each data set, we determined the mean amount of intra- and interspecific aggregation and three types of within-sample or local species diversity: species richness, evenness, and dominance. Using regression, we found a negative relationship between intraspecific aggregation and all three types of local diversity. Intraspecific aggregation explained a significant percentage of the variation in species diversity, typically between $20 \%$ and $60 \%$. By comparison, interspecific aggregation usually explained $<1 \%$ of the variation in species diversity. Our study provides empirical support for the species-aggregation relationship as a general macroecological pattern that emerges from intraspecific aggregation. sity.

Key words: arthropods; intraspecific aggregation; macroecology; neutral model; species diver-
\end{abstract}

\section{INTRODUCTION}

The individuals of most species commonly occur in aggregated patterns more often than in regular or random patterns. That is, many species exhibit intraspecific aggregation (Taylor et al. 1978). Numerous ecologists have sought to link patterns of species diversity to intraspecific aggregation patterns (see Ives 1991, Shorrocks and Sevenster 1995, Wertheim et al. 2000). Most recently, Stoll and Prati (2001) tested and found support for spatial competition models that invoke intraspecific aggregation to explain coexistence and diversity in plant communities. Previous to that, Ives (1988) developed the aggregation model of species coexistence to explain the diversity of insect species that use discrete resource patches (Atkinson and Shorrocks 1981, Hanski 1981).

The aggregation model states that the coexistence of some insect species is enhanced by intraspecific ag-

Manuscript received 3 October 2002; revised 14 February 2003; accepted 18 February 2003; final version received 7 April 2003. Corresponding Editor: F. He.

${ }^{1}$ Present address: Department of Fishery and Wildlife Biology, Colorado State University, Fort Collins, Colorado 80523-1474 USA.

${ }^{2}$ Present address: Environmental Science and Policy Program, Drake University, Des Moines, Iowa 50311 USA. gregation among a limited number of resource patches that are used for copulation, oviposition, and larval development (Shorrocks et al. 1984, Shorrocks and Rosewell 1986, Ives 1988, 1991, Jaenike and James 1991, Sevenster 1996, Sevenster and Van Alphen 1996, Palestrini et al. 1998, Krijger and Sevenster 2001, Woodcock et al. 2002). Because of intraspecific aggregation, larvae compete with conspecifics more so than heterospecifics and competitive exclusion is avoided (Atkinson and Shorrocks 1981, Hanski 1981, Ives and May 1985, Ives 1988). Thus, intraspecific aggregation increases the total species diversity found throughout all resource patches. In contrast to this prediction of total species diversity, however, little has been known about the correlation between diversity within resource patches and intraspecific aggregation.

In a recent theoretical study, He and Legendre (2002) derived a similar prediction. Their species-aggregation relationship describes how intraspecific aggregation decreases the number of species expected in small samples from some larger geographic area. The speciesaggregation relationship is a general formulation of how intraspecific aggregation affects local or withinsample species diversity. Unlike the aggregation model, it does not require a specific mechanism (e.g., resource competition) and it is not taxon specific (e.g., 
aggregation model has mostly been applied to carrion flies, fruit flies, and dung beetles). The species-aggregation relationship is presented in the same general terms as is the species-area relationship ( $\mathrm{He}$ and Legendre 2002).

The goal of our study was to use empirical data to determine whether a decrease in species diversity within sampling sites emerges as a general consequence of increasing intraspecific aggregation among the sampling sites. We tested for significant effects of intraspecific aggregation on species richness, evenness, and dominance (collectively referred to as species diversity). "Evenness" as used here refers to the relative equality of species abundances, not to the degree to which a single species is evenly distributed among sites. We compiled species distribution-abundance data sets (each one a separate species assemblage) into a set of metadata which we analyzed using regression. Each data set consisted of species and individuals distributed among spatially distinct "sites" or "samples." For each data set, we calculated the mean amount of intraspecific aggregation. We predicted that an increase in mean intraspecific aggregation should correspond to a decrease in within-sample diversity, in accord with the species-aggregation relationship ( $\mathrm{He}$ and Legendre 2002) and the aggregation model (Ives 1988). For comparison, we also estimated interspecific aggregation for each data set and tested for a relationship between it and within-sample diversity.

\section{Methods}

\section{Compilation of data sets}

We collected 76 data sets of the distribution and abundance of arthropod species (see the Appendix). Most were published in ecological, entomological, or conservation journals. We included only those studies that contained distribution and abundance data for adult individuals of at least 15 species occurring among three or more sampling "sites." The spatial scale of the data sets varied considerably. At the finest spatial scale, sampling sites were separated from one another by at least $50 \mathrm{~m}$ but no more than $2 \mathrm{~km}$; often these were samples (e.g., pitfall traps) arranged on a grid or along a transect. At an intermediate spatial scale, sites were separated from one another by at least $2 \mathrm{~km}$ but no two sites were $>100 \mathrm{~km}$ distant. At the broadest scale, sites were at least $50 \mathrm{~km}$ apart and at least a few sites were more than $100 \mathrm{~km}$ distant. We included only studies in which sampling effort (i.e., sampling duration) was relatively consistent among sample locations. The most common sampling methods were pitfall trapping, flight-intercept trapping, insecticide fogging, blacklighting, sweep-netting, vacuum-suctioning, leaf-litter collection, and visual observation (see Appendix). Bee- tles, ants, butterflies, moths, and spiders were the most common arthropod groups represented among the studies. For each data set, we measured the mean amount of intra- and interspecific aggregation and the mean species diversity of the sites.

\section{Measurement of intraspecific aggregation}

We measured the intraspecific aggregation of each non-singleton species (abundance $>1$ ) using the $J$ index of Ives $(1988,1991)$ :

$$
J=\left\{\left[\frac{\sum x_{j}\left(x_{j}-1\right)}{X N}\right]-X\right\} \frac{1}{X}
$$

where $x_{j}$ is the number of individuals at site $j, X$ is the mean number of individuals per site, and $N$ is the number of sites. If individuals are independently and randomly dispersed among the sites then the number of individuals per site follows a Poisson distribution. Each individual exists at its site (or in its sample) with some number of conspecifics. Averaging across all individuals, $J$ measures the proportional increase in the mean number of conspecifics present with each individual in the sample relative to the mean number expected based on a Poisson distribution (Ives 1988, 1991). For example, $J=1$ represents an increase of $100 \%$ (or a doubling) in the mean number of conspecifics present along with each individual in the same sample. When $J=0$, individuals are randomly distributed whereas values of $J>0$ represent an increasing amount of intraspecific aggregation. $J$ is a mean over individuals, not samples.

$J$ is an unbiased estimator (Ives 1988, 1991) and has been extensively used in the theoretical development and testing of the aggregation model of coexistence (Ives 1991, Giller and Doube 1994, Shorrocks and Sevenster 1995, Sevenster 1996, Sevenster and Van Alphen 1996, Palestrini et al. 1998, Morand et al. 1999). $J$ is related to the clumping parameter, $k$, of the negative binomial distribution in the form of $J=1 / k$. One can fit $k$ to a species' distribution among a set of samples or sites so as to estimate intraspecific aggregation. In general, $k$ is affected by the number of samples, size of sample plots, and species abundance (Krebs 1999), so would be $J$. We also measured intraspecific aggregation using the standardized version of the Morisita index that is considered to be one of the most reliable measures of its kind (Krebs 1999). Results derived from the standardized Morisita index were nearly identical to those obtained using $J$, so we present results for the latter only.

\section{Measurement of interspecific aggregation}

For each data set, we used the $C$ index of Ives (1988, 1991) to measure the interspecific aggregation of each species. As originally presented by Ives (1988), C mea- 
sures the amount of association between two species; it is a pairwise metric. We modified Ives's formula so that $C$ would measure the tendency of individuals of a species to aggregate with individuals of all other species (i.e., the superspecies approach of Shorrocks and Rosewell 1986, Wertheim et al. 2000, Krijger and Sevenster 2001):

$$
C=\left\{\left[\frac{\sum x_{j} h_{j}}{X N}\right]-H\right\} \frac{1}{H}
$$

where $h_{j}$ is the number of heterospecific individuals at site $j$ and $H$ is the mean number over all sites. An increasingly positive value of $C$ represents increasing interspecific aggregation whereas an increasingly negative value represents interspecific repulsion. For each species, $C$ measures the proportional increase in the average number of heterospecifics present with each individual in the sample relative to the number expected based on a Poisson distribution. As with $J, C$ is averaged over individuals not sampling sites. We used the mean $C$ of species in each data set in the regression analyses. We also measured interspecific aggregation using a metric based on Schluter's (1984) V ratio. We obtained the same results as produced by the $C$ index thus results for $V$ are not presented.

\section{Measurement of species diversity}

For each data set, we measured the mean diversity within sites using species richness, species evenness (Shannon index), and species dominance (Simpson index or Gini coefficient). The "contribution" of each site to the mean was weighted by the number of individuals recorded at the site. That is, the mean was obtained as $\sum d_{j} q_{j}$ for all sites $j=1$ to $N$, where $q_{j}$ is the number of individuals at site $j$ as a proportion of the total number of individuals at all sites and $d_{j}$ is the diversity estimate at that site as measured by richness, the Shannon index, or Simpson index. Species richness is the number of species at a site whereas the Shannon and Simpson indices measure diversity based on the proportional abundances of species at a site. The Shannon index is determined as $-\Sigma p_{i} \ln \left(p_{i}\right)$ and the Simpson index as $1-\Sigma p_{i}^{2}$ where $p_{i}$ is the proportional abundance of species $i$ for $i=1$ to $S$ species at the sampling site. The Shannon and Simpson indices assess different aspects of diversity than does species richness. More precisely the Shannon index measures evenness or the degree to which species are equally represented at a site. The Simpson index measures the degree to which one or a few species are numerically dominant. For each diversity metric, we also calculated the total diversity found in each data set by pooling the data of all sites (of a data set) into a single sample. This then allowed us to express the mean diversity within the sites of a data set (i.e., mean local diversity) as a percentage of the total diversity. Note that the mean local diversity could not exceed the total diversity because we were using a weighted average as explained above (Lande 1996).

\section{Regression analyses}

Each of the 76 data sets together comprised a set of metadata that was analyzed using multiple linear regression. We performed a set of regressions in which species richness, evenness, or dominance was always the dependent variable while intra- or interspecific aggregation was always the independent variable along with number of sites, number of species, and total number of individuals in a data set. These latter three variables were considered sampling artifacts given that they mostly derived from sampling design and effort and not from any biological or ecological process. The data sets exhibited substantial variation in these sampling variables, and preliminary analyses indicated that they were often significantly correlated with the diversity and aggregation variables. Therefore, we used multiple regression to control the effects of the sampling variables (species number and total number of individuals were log transformed). Multiple regression allowed us to obtain the partial regression coefficients for the aggregation variables. Those coefficients assessed the effect of aggregation on diversity in the absence of effects due to the sampling variables. This is one of many common uses of multiple regression (Philippi 1993, Sokal and Rohlf 1995) and is equivalent to removing the effects of nuisance variables by performing regression on their residuals (Hayes and Shonkwiler 1996).

Except for the number of sites, all of the independent variables probably included some measurement error. Error in the measurement of independent variables can lead to incorrect estimation of regression coefficients, including changes in sign, and dubious $P$ values (Pedhazur 1997). These unwanted effects of measurement error are most pronounced when the variance in measurement error is relatively large compared to the total variance which includes measurement error, sampling error, and natural variation. That is, when the reliability of a variable ( $1-$ [variance in measurement error/total variance]) is high, say $>0.75$, measurement error is relatively small. In such cases, measurement error has negligible effects on the regression coefficients, particularly if the regression coefficients are large and highly significant (Pedhazur 1997).

We assumed that the measurement error in each of our independent variables was small enough as to not affect the significance or sign of our partial regression coefficients. To verify this assumption we simulated measurement error in each independent variable (ex- 
cept number of sites) for each regression analysis (see Results). We created simulated data to represent the "true" (without measurement error) values of the independent variables and then performed multiple regression on the simulated data. This was repeated 100 times for each regression analysis. In the simulations for producing the "true" data, we assumed that the observed data underestimated the number of species and number of individuals of each data set. "True" values for number of species and number of individuals were randomly set to be between 1.5 and 2 times the observed values. We assumed that intra- and interspecific aggregation could be either over- or underestimated, thus "true" values for these variables were randomly set to be between half and twice the observed values. These randomizations resulted in reliability values between 0.16 and 0.66 for intraspecific aggregation, 0.19 and 0.93 for interspecific aggregation, 0.19 and 0.54 for number of species, and 0.19 to 0.60 for number of individuals. By simulating "true" values we also simulated measurement error in the observed values; however, the simulation had to be constrained such that the variance in measurement error was less than the total variance observed; i.e., reliability must be between 0 and 1 .

For each diversity metric, we conducted a multiple regression using either intra- or interspecific aggregation ( $J$ and $C$ respectively) and the three sampling variables, with 76 total observations representing the 76 data sets. To control for taxon-related variation, we performed separate regressions on the ground-dwelling beetle data sets $(n=28)$, and the butterfly/moth data sets $(n=14)$. Given that small spatial scales are probably most appropriate for testing the species-aggregation relationship (He and Legendre 2002; F. He, personal communication), we also performed separate regressions on those data sets representing the fine spatial scale $(n=17)$.

\section{RESULTS}

There was substantial variation among the 76 data sets in mean intraspecific aggregation $(J$ ranged from -0.13 to 8.79 ) and mean interspecific aggregation $(C$ ranged from -0.25 to 2.85 ). Likewise, mean local species diversity as a percentage of total diversity varied substantially: range of species richness, $13-85 \%$; range of the Shannon index, 36-96\%; and range of the Simpson index, 38-99\%. Most importantly, there was enough variation in each of the aggregation variables to provide a meaningful test of the effect of each on the three diversity variables which were normally distributed. As predicted, the mean amount of intraspecific aggregation had a significant negative effect on all three types of species diversity. All of the partial regression coefficients for $J$ regressed against the diver-
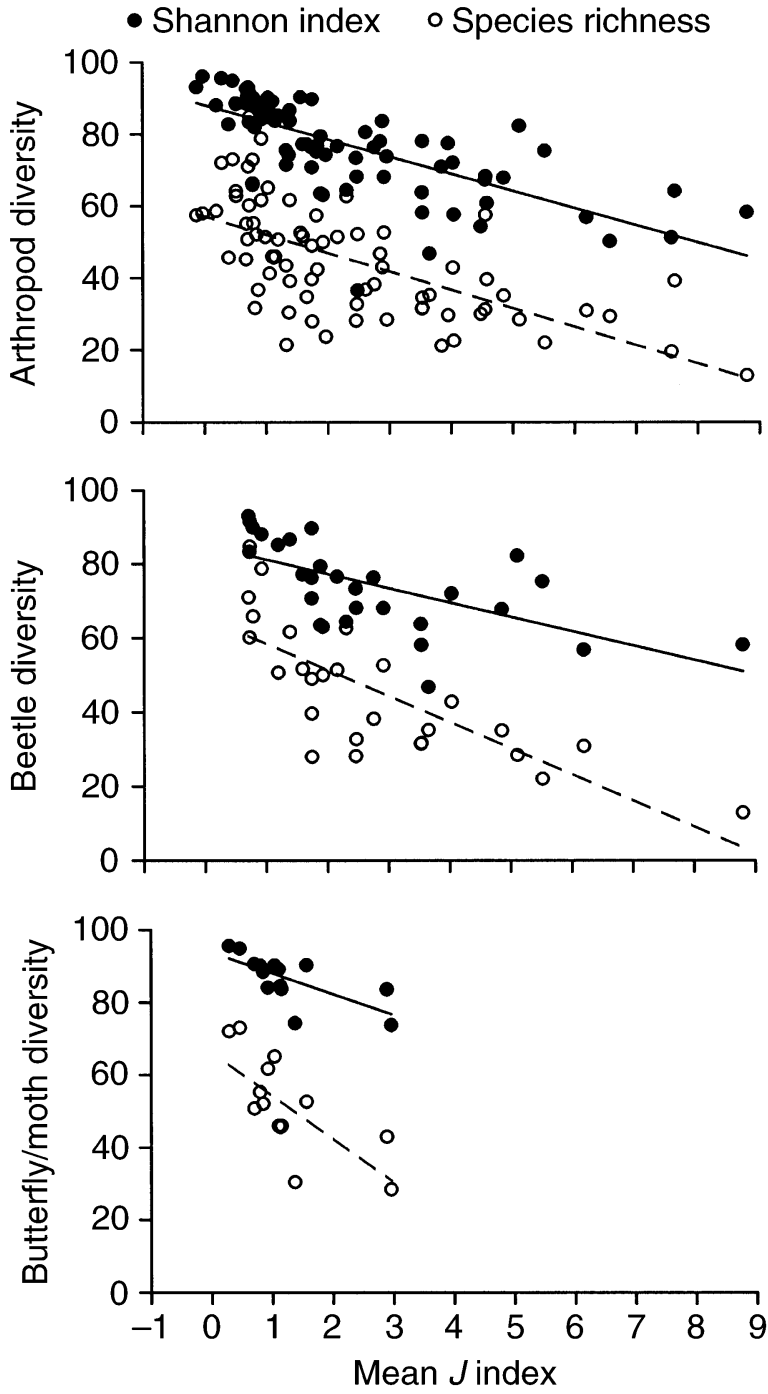

FIG. 1. The negative relationship between intraspecific aggregation (mean $J$ of species in an assemblage) and mean within-sample species richness and Shannon index for 76 arthropod assemblages, 28 beetle assemblages, and 14 butterfly/moth assemblages. Mean species diversity is given as a percentage of total diversity. Species dominance (Simpson index) exhibited a decrease very similar to that for the Shannon index. Lines indicate a relationship in which the partial regression coefficient from a multiple regression analysis is significant $(P<0.05)$

sity metrics were negative and either slightly significant $(P<0.05)$ or highly significant $(P<0.00001)$. Plots of the univariate relationships also exhibited decreasing species diversity with increasing intraspecific aggregation (Fig. 1). We did not find significant relationships between interspecific aggregation and species richness, evenness, or dominance.

The coefficients of determination ( $R^{2}$ values $)$ derived from the multiple regression models provided estimates 
TABle 1. Percentage of variation in species diversity explained by intra- and interspecific aggregation and sampling variables.

\begin{tabular}{|c|c|c|c|}
\hline \multirow[b]{2}{*}{ Variable } & \multicolumn{3}{|c|}{ Species diversity } \\
\hline & Richness & Evenness & Dominance \\
\hline \multicolumn{4}{|l|}{ All arthropod data sets $(n=76)$} \\
\hline Sampling variables & 50.7 & 27.3 & 44.8 \\
\hline Intraspecific aggregation $(J)$ & $15.7 * * * *$ & $34.4 * * * *$ & $13.9^{*}$ \\
\hline Interspecific aggregation $(C)$ & $\ll 1.0$ & $\ll 1.0$ & $\ll 1.0$ \\
\hline \multicolumn{4}{|l|}{ Beetle data sets $(n=28)$} \\
\hline Sampling variables & 54.7 & 10.6 & 34.8 \\
\hline Intraspecific aggregation $(J)$ & $20.6^{* * *} *$ & $54.3 * * * *$ & $23.6^{*}$ \\
\hline Interspecific aggregation $(C)$ & $<1.0$ & $\ll 1.0$ & $\ll 1.0$ \\
\hline \multicolumn{4}{|l|}{ Butterfly/moth data sets $(n=14)$} \\
\hline Sampling variables & 35.4 & 23.8 & 30.1 \\
\hline Intraspecific aggregation $(J)$ & $35.3 *$ & $31.6^{*}$ & $20.2 \dagger$ \\
\hline Interspecific aggregation $(C)$ & 4.2 & $<1.0$ & 3.0 \\
\hline \multicolumn{4}{|l|}{ Fine-scale data sets $(n=17)$} \\
\hline Sampling variables & 74.4 & 31.1 & 48.0 \\
\hline Intraspecific aggregation $(J)$ & $5.3 \dagger$ & $24.1 *$ & $15.1 *$ \\
\hline Interspecific aggregation $(C)$ & 1.0 & 12.7 & 13.0 \\
\hline
\end{tabular}

of the percent of variation in the diversity metrics explained by variation in mean intraspecific aggregation. Of the 76 data sets representing arthropod assemblages, intraspecific aggregation explained $15.7 \%$ of the variation in mean species richness (of sampling sites) of a data set, $34.4 \%$ of variation in evenness, and $13.9 \%$ of variation in dominance (Table 1). Much less of the variation $(\ll 1 \%)$ in diversity was explained by interspecific aggregation.

We conducted separate regressions on the subset of data sets consisting of only ground-dwelling beetles (mostly Carabidae, Staphylinidae, and Tenebrionidae) and the subset consisting of only moths and butterflies. Again, local species diversity declined with increasing intraspecific aggregation (Fig. 1) and a greater amount of variation in richness, evenness, and dominance, was explained by intraspecific aggregation than interspecific aggregation (Table 1 ). When the multiple regression analysis was limited to just the fine-scale data sets (those in which separation between sites is $<2 \mathrm{~km}$ ), intraspecific aggregation explained more variation in diversity than did interspecific aggregation (Table 1). As predicted, local species diversity declined with increasing intraspecific aggregation at this scale (Fig. 2).

Finally, the negative relationships between mean intraspecific aggregation and local species diversity appeared to be robust to error in the measurement of the independent variables. That is, even when the independent variables were assumed to have measurement error, the partial regression coefficients always re- mained negative and usually significant (Table 2). The only exception was the analysis of the butterfly and moth data sets; if we assume measurement error then the observed relationships between intraspecific ag-

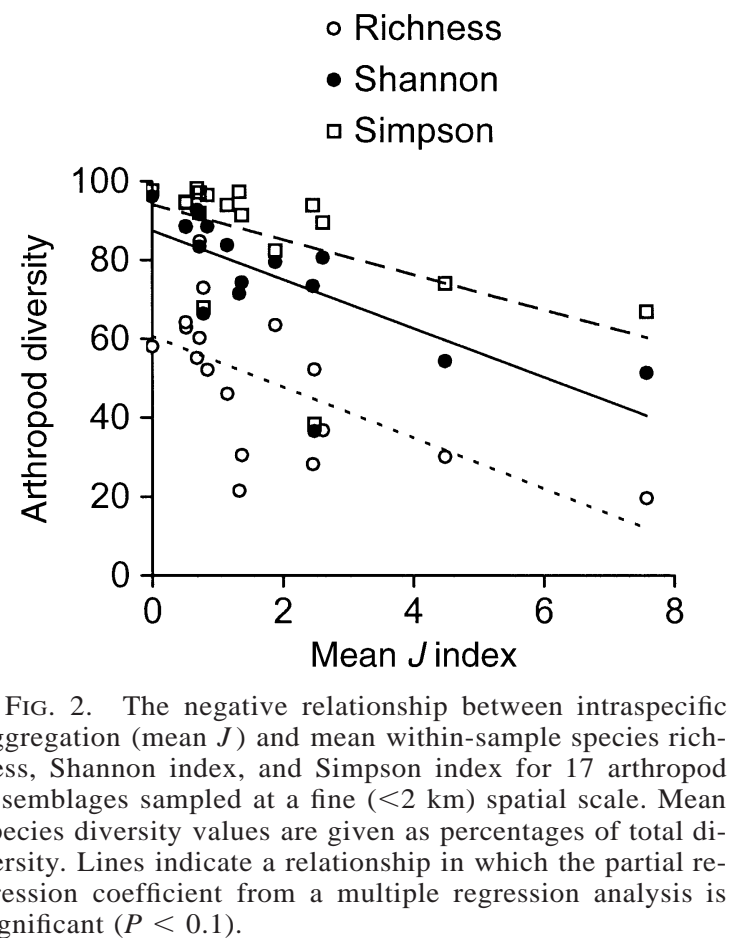

- Shannon

Simpson

FIG. 2. The negative relationship between intraspecific aggregation (mean $J$ ) and mean within-sample species richness, Shannon index, and Simpson index for 17 arthropod assemblages sampled at a fine $(<2 \mathrm{~km})$ spatial scale. Mean versity. Lines indicate a relationship in which the partial regression coefficient from a multiple regression analysis is significant $(P<0.1)$ 
TABLE 2. The observed partial regression coefficients for intraspecific aggregation regressed against each diversity metric (richness, evenness, and dominance) and the simulated range of possible values of the coefficients assuming error in the measurement of all independent variables.

\begin{tabular}{|c|c|c|c|}
\hline Metric & $\begin{array}{c}\text { Partial regression } \\
\text { coefficient } \dagger\end{array}$ & $\begin{array}{l}\text { Range assuming } \\
\text { measurement error }\end{array}$ & $\begin{array}{l}\text { Percent significant } \\
\quad \text { at } P=0.05 \ddagger\end{array}$ \\
\hline \multicolumn{4}{|c|}{ All arthropod data sets } \\
\hline Richness & -4.5 & -3.6 to -1.5 & 100 \\
\hline Evenness & -5.5 & -3.9 to -2.2 & 100 \\
\hline Dominance & -3.1 & -2.3 to -1.2 & 100 \\
\hline \multicolumn{4}{|l|}{ Beetle data sets } \\
\hline Richness & -5.2 & -5.9 to -2.8 & 100 \\
\hline Evenness & -5.6 & -5.2 to -2.7 & 100 \\
\hline Dominance & -3.2 & -3.6 to -1.5 & 98 \\
\hline \multicolumn{4}{|c|}{ Butterfly/moth data sets } \\
\hline Richness & -12.4 & -17.4 to -3.9 & 60 \\
\hline Evenness & -5.7 & -8.5 to -1.2 & 44 \\
\hline Dominance & -3.1 & -5.5 to -0.1 & 40 \\
\hline \multicolumn{4}{|c|}{ Fine-scale data sets } \\
\hline Richness & -3.9 & -9.8 to -3.9 & 100 \\
\hline Evenness & -7.6 & -11.6 to -2.6 & 88 \\
\hline Dominance & -5.8 & -8.6 to -1.5 & 50 \\
\hline
\end{tabular}

$\uparrow$ See Table 1 for significance of observed partial regression coefficients.

\$ The percentage of simulated regression coefficients that were significant.

gregation and local diversity for those data sets may not be significant (Table 2).

\section{DisCUSSION}

Analyzing a variety of arthropod assemblages, we found support for the species-aggregation relationship (He and Legendre 2002), a decrease in local species diversity with increasing intraspecific aggregation. This inverse relationship between intraspecific aggregation and species diversity appears to be very general. Together, our compiled data sets represented several different arthropod taxa inhabiting sampling sites at a wide range of spatial scales, habitats, and biomes. In addition, the total richness of the arthropod assemblages varied substantially from one data set to the next. We conducted regression analyses on several subsets of data and always uncovered the same pattern: decreasing local diversity coupled to increasing intraspecific aggregation (Fig. 1). Indeed, intraspecific aggregation was capable of explaining a large amount of the variation in mean species diversity, particularly when beetle assemblages were analyzed separately from the butterfly-moth assemblages (Table 1).

Perhaps, of most importance, we also found the predicted negative relationship between intraspecific aggregation and local diversity from the analysis of the fine-scale data sets (Fig. 2). The species-aggregation relationship (He and Legendre 2002) was developed, in part, by analysis of the spatial distribution of tropical trees in 50-ha plots. Our study provides evidence of the relationship at a similar scale, but in a very different group of organisms.

Despite all the potential sources of variation (e.g., differences in spatial scale, habitat, etc.) among the data sets, the amount of variation in species diversity (richness, evenness, and dominance) explained by intraspecific aggregation was substantially larger than the amount explained by interspecific aggregation (Table 1). Interestingly, most species did exhibit interspecific aggregation; i.e., mean $C$ was positive for most data sets and $C$ values for individual species were typically positive. Therefore, we can conclude that although arthropod individuals tend to congregate with heterospecifics, this interspecific aggregation has little effect on local species diversity.

The greater effect of intraspecific compared to interspecific aggregation on local species diversity agrees with recent theory derived from neutral models. In neutral models, the competitive abilities of individuals are neutral with regard to their species identity, though individuals may still compete and interact in other ways (Hubbell 2001) or not at all (Caswell 1976). Neutral theory predicts that the diversity within local ecological communities can be explained by the dispersal capacities and intraspecific aggregation tendencies of species that are constituents of some larger regional species pool (Hubbell 2001, Bell 2001). Hence, it may not be necessary to invoke species interactions as mechanisms that influence local diversity, even if those interactions do exist. If we assume that species inter- 
actions exist in the species assemblages (data sets) of our study then we can conclude that these interactions, as roughly measured by the $C$ index, appear to have less of an influence on local species diversity than do the mechanisms determining patterns of intraspecific aggregation. Alternatively, arthropod species may be noninteractive (Shorrocks et al. 1984, Strong et al. 1984) or the $C$ index may be a poor indicator of species interactions, such that the present study is not an adequate test of the effect of species interactions on local arthropod diversity.

With this study, we have provided further evidence that increasing intraspecific aggregation can substantially decrease local species diversity (He and Legendre 2002). We have not discussed the mechanisms that lead to intraspecific aggregation partly because the data do not allow us to test mechanisms and partly because the causes of intraspecific aggregation are numerous and varied. Conspecifics might aggregate because of similar habitat requirements, a shared resource base, to find a mate, avoid a predator, or for various other reasons. Indeed, we suggest that an attractive aspect of the species-aggregation relationship (He and Legendre 2002) is that it does not invoke a specific process to directly explain species diversity. Similarly the species-area relationship can be described without invoking a specific process and likewise has several potential explanations. Explanations for the species-aggregation relationship should be developed and tested; however the actual description of the relationship need not refer to mechanism to be useful and predictive.

The relationship between intraspecific aggregation and species diversity deserves further study. The pattern itself needs to be more thoroughly analyzed and documented just as the species-area relationship has been widely documented in the last half century. Processes that lead to intraspecific aggregation need to be studied under the premise that they may also affect species diversity. Perhaps, a more comprehensive understanding of species diversity can be obtained by establishing the generality and identifying the causes of a few relatively simple ecological relationships, including the species-aggregation relationship.

\section{ACKNOWLEDGMENTS}

We thank Kohji Yamamura, Eric Porter, and an anonymous reviewer for comments and suggestions that improved the manuscript. The following people kindly supplied us with unpublished data sets: R. Anderson (Canadian Museum of Nature, Ottawa), C. Buddle (McGill University), C. Carleton (Louisiana State University), M. Cigliano (Argentina), M. Di Giulio (Switzerland), M. Eyre (United Kingdom), A. Floren (Germany), K. Gandhi (University of Alberta), J. Gering (Truman State University), T. Magura (Hungary), P. Martikainen (Finland), J. Spence (University of Alberta), and S. Torrusio (Argentina).

\section{Literature Cited}

Atkinson, W. D., and B. Shorrocks. 1981. Competition on a divided and emphemeral resource: a simulation model. Journal of Animal Ecology 50:461-471.

Bell, G. 2001. Ecology-neutral macroecology. Science 293:2413-2418.

Caswell, H. 1976. Community structure: a neutral model analysis. Ecological Monographs 46:327-354.

Giller, P. S., and B. M. Doube. 1994. Spatial and temporal cooccurence of competitors in southern African dung beetle communities. Journal of Animal Ecology 63:629-643.

Hanski, I. 1981. Coexistence of competitors in patchy environments with and without predation. Oikos 37:306312.

Hayes, J. P., and J. S. Shonkwiler. 1996. Analyzing massindependent data. Physiological Zoology 69:974-980.

He, F. L., and P. Legendre. 2002. Species diversity patterns derived from species-area models. Ecology 83:11851198.

Hubbell, S. P. 2001. The unified neutral theory of biodiversity and biogeography. Princeton University Press, Princeton, New Jersey, USA.

Ives, A. R. 1988. Aggregation and the coexistence of competitors. Annales Zoologici Fennici 25:75-88.

Ives, A. R. 1991. Aggregation and coexistence in a carrion fly community. Ecological Monographs 61:75-94.

Ives, A. R., and R. M. May. 1985. Competition within and between species in a patchy environment: relations between microscopic and macroscopic models. Journal of Theoretical Biology 115:65-92.

Jaenike, J., and A. C. James. 1991. Aggregation and the coexistence of mycophagous Drosophila. Journal of Animal Ecology 60:913-928.

Krebs, C. J. 1999. Ecological methodology. Second edition. Addison-Wesley Educational Publishers, Menlo Park, California, USA.

Krijger, C. L., and J. G. Sevenster. 2001. Higher species diversity explained by stronger spatial aggregation across six neotropical Drosophila communities. Ecology Letters 4:106-115.

Lande, R. 1996. Statistics and partitioning of species diversity, and similarity among multiple communities. Oikos 76:5-13.

Morand, S., R. Poulin, K. Rohde, and C. Hayward. 1999. Aggregation and species coexistence of ectoparasites of marine fishes. International Journal of Parasitology 29: 663-672.

Palestrini, C., E. Barbero, and A. Rolando. 1998. Intra- and interspecific aggregation among dune beetles (Coleoptera: Scarabaeoidea) in an Alpine pasture. Journal of Zoology 245:101-109.

Pedhazur, E. J. 1997. Multiple regression in behavioral research. Third edition. Harcourt Brace Publishing, Orlando, Florida, USA.

Philippi, T. E. 1993. Multiple regression: herbivory. Pages 183-210 in S. E. Scheiner and J. Gurevitch, editors. Design and analysis of ecological experiments. Chapman and Hall, New York, New York, USA.

Schluter, D. 1984. A variance test for detecting species associations, with some example applications. Ecology 65: 998-1005.

Sevenster, J. G. 1996. Aggregation and coexistence. I. Theory and analysis. Journal of Animal Ecology 65:297-307.

Sevenster, J. G., and J. J. M. Van Alphen. 1996. Aggregation and coexistence. II. A neotropical Drosophila community. Journal of Animal Ecology 65:308-324. 
Shorrocks, B., and J. Rosewell. 1986. Guild size in drosophilids: a simulation model. Journal of Animal Ecology 55 527-541.

Shorrocks, B., J. Rosewell, K. Edwards, and W. D. Atkinson. 1984. Interspecific competition is not a major organizing force in many insect communities. Nature 310:310-312.

Shorrocks, B., and J. G. Sevenster. 1995. Explaining local species diversity. Proceedings of the Royal Society of London Series B 260:305-309.

Sokal, R. R., and F. J. Rohlf. 1995. Biometry. Third edition. Freeman, New York, New York, USA.

Stoll, P., and D. Prati. 2001. Intraspecific aggregation alters competitive interactions in experimental plant communities. Ecology 82:319-327.
Strong, D. R., J. H. Lawton, and T. R. E. Southwood. 1984. Insects on plants: community patterns and mechanisms. Blackwell Publishing, Oxford, UK.

Taylor, L. R., I. P. Woiwood, and J. N. Perry. 1978. The density-dependence of spatial behavior and the rarity of randomness. Journal of Animal Ecology 47:383-406.

Wertheim, B., J. G. Sevenster, I. E. M. Eijs, and J. J. M. Van Alphen. 2000. Species diversity in a mycophagous insect community: the case of spatial aggregation vs. resource partitioning. Journal of Animal Ecology 69:335-351.

Woodcock, B. A., A. D. Watt, and S. R. Leather. 2002. Aggregation, habitat quality and coexistence: a case study on carrion fly communities in slug cadavers. Journal of Animal Ecology 71:131-140.

\section{APPENDIX}

Sources and descriptions of the arthropod data sets used in this study are available in ESA's Electronic Data Archive: Ecological Archives E084-092-A1. 